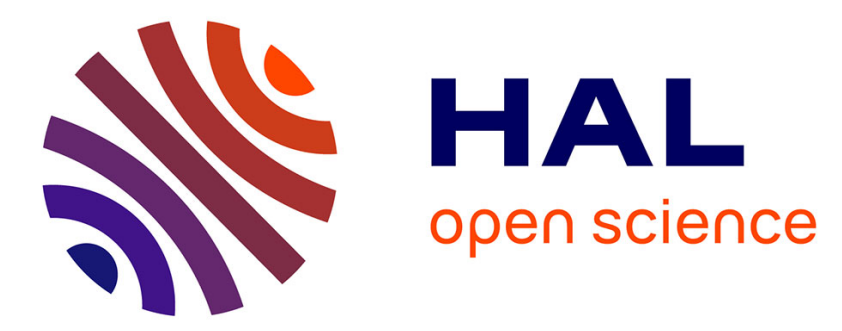

\title{
Quantitative risk analysis in radiotherapy using Bayesian networks
}

Alexandre Reitz, Eric Levrat, Jean-François Pétin

\section{To cite this version:}

Alexandre Reitz, Eric Levrat, Jean-François Pétin. Quantitative risk analysis in radiotherapy using Bayesian networks. Annual Conference of the European Safety and Reliability Conference, ESREL 2013, Aug 2013, Amsterdam, Netherlands. pp.520. hal-00880774

\section{HAL Id: hal-00880774 https://hal.science/hal-00880774}

Submitted on 6 Nov 2013

HAL is a multi-disciplinary open access archive for the deposit and dissemination of scientific research documents, whether they are published or not. The documents may come from teaching and research institutions in France or abroad, or from public or private research centers.
L'archive ouverte pluridisciplinaire HAL, est destinée au dépôt et à la diffusion de documents scientifiques de niveau recherche, publiés ou non, émanant des établissements d'enseignement et de recherche français ou étrangers, des laboratoires publics ou privés. 


\title{
Quantitative risk analysis in radiotherapy using Bayesian networks
}

\author{
A. Reitz, E. Levrat, J-F. Pétin \\ Lorraine University, Research Centre for Automatic Control (CRAN) $\square$ CNRS UMR 7039
}

\begin{abstract}
Radiotherapy is a complex process, relying on different human skills and highly technical devices, which consist in exposing tumors to ionizing rays. Radiotherapy is composed of sessions which are repeated 3 to 5 times a week during several weeks. A recent study of WHO concerning risks in radiotherapy points out that only half of all incidents declared since 30 years are due to technical causes, the other half being due to human and organizational causes.

To improve patient safety, our proposal considers these three dimensions (technical, human and organizational) in a global assessment of risks (over-irradiation and under-irradiation) incurred by the patients during radiotherapy and the impact of the existing safety barriers. Our previous works focused on qualitative analysis through functional, dysfunctional, and organizational analysis. These studies proposed systematic guidelines using formalisms such as SADT or FMEA/HAZOP to identify dysfunctional relations inside and between each dimension, and their impact of incurred risks.
\end{abstract}

This paper aims to unify all these qualitative models in a probabilistic relational model that enables to provide not only qualitative recommendations but also a quantitative evaluation of risk. Radiotherapy can be seen as a product lifecycle, by considering treatment parameters as a product designed by a multidisciplinary team, realized and validated during the first treatment session, and finally used for each radiotherapy session. Each of these main phases are modeled using a Bayesian network pattern (derived from cognitive engineering) that captures the different causal influences of activity inputs (technical but also organizational) with regards to the activity failure modes and outputs. These Bayesian elementary networks are aggregated according to the causal flows identified in the previous qualitative analysis. Expert knowledge and adverse event databases should be used to parameter the network and provide the expected risk evaluation for each medical center.

Radiotherapy patient process is a complex sociotechnical system, involving high-end medical devices and techniques, used by highly skilled persons. Several years ago, radiotherapy had to face accidents and have modified treatment process to make it safer. Those macroscopic changes have had beneficial effects, but cannot prevent punctual incidents. In this context, our proposal considers technical, human, and organizational dimensions in a global assessment of punctual over-irradiation and under-irradiation incurred by the patients during radiotherapy and the impact of the existing safety barriers.

In this paper is presented the unification of previous qualitative models (Section 2) in a probabilistic relational model (Bayesian Networks) that enables to provide not only qualitative recommendations but also a quantitative evaluation of patient risks, and the impact of barriers on these risks. For this, a modeling method based on probabilistic fault tree is used to obtain a first macroscopic model structure of risk in radiotherapy (Section $3.2 \& 3.3$ ). On this base, a second model considering safety barriers is proposed (Section 3.3). Finally, a quantitative comparison between these models based on the same set of data is presented.

\section{PROBLEM}

Radiotherapy principle consists in exposing tumors to ionizing rays. To preserve surrounding organs and tissues, this exposure is divided in dose fractions. Each fraction is distributed over a set of beams aiming tu- 
mor with different incidence angles. Each set is distributed in a treatment session, a radiotherapy treatment is composed of several session repeated 5 times a week until total dose is distributed.

A radiotherapy treatment is a complex sociotechnical system (Aloui 2008). Indeed, to implement a radiotherapy treatment, therapist oncologists, medical physicist and dosimetrists have to find the best way to use highly technical medical devices to treat each patient, by designing (and using) a set of parameters called treatment plan.

That kind of cancer treatment can generate risks to patient:

- Over irradiation of healthy organs or tumor, which can lead to new diseases,

- Under irradiation of tumor, signifying ineffective treatment.

In year 2011 in France, more than 160,000 Patients were treated using radiotherapy (more than 185,000 treatments) (INCa 2013). Only 4 incidents concerning exposure of patients to the studied risk (over irradiation, under irradiation) were declared in the same period to French Nuclear Safety Agency (ASN). Regarding this rate, Radiotherapy process looks safe, but:

- Each adverse event concerns over irradiation or under irradiation during several consecutive sessions, called significant events, which may have direct pathogenic effects to patient. Punctual over irradiation or under irradiation have no direct effects so they are not declared as incidents to Safety Nuclear Agency (Birraux 2013);

- Radiotherapy centers were equipped several years ago with security and safety devices that detect and alert in case of deviations on treatment process. These devices allow detecting and catching adverse situation before the occurrence of incident.

If we look at international reporting systems (Radiation Oncology Safety Information System - ROSIS, Safety in Radiation Oncology - SAFRON), or radiotherapy centers adverse event databases, punctual over /under Irradiation and near events that can lead to it are more numerous. They are generally caught up by reengineering treatment plan.

For each event declared to ROSIS reporting system or for the four adverse events declared to ASN, appears that they are mostly detected during treatment sessions (40\% of ROSIS base). This means that a succession of errors occurred during previous treatment activities, and during safety activities of the process (Holmberg 2012). Process is not safe as it seems, and consequences of significant events are serious for patients, radiotherapy practitioners and for care facilities (Epinal trial, 2007).

A recent study (French Health high authority - HAS 2013) shows that half of all radiotherapy events (46\%) are initiate during radiotherapy setup step where patient treatment plan is designed, and are non-detected during first treatment session where patient treatment plan is validate. On the other hand, occurrence of risks in radiotherapy was studied by (World Health Organization 2008), and points out that only half of all incidents declared since 30 years are due to technical causes, the other half being due to human and organizational causes. ROSIS and ASN declaration forms register those different roots for each new event. Trying to systematically detect causes of punctual event should make process safer. Our work aims to bring better knowledge on radiotherapy process, and existing barriers effectiveness.

In this context, our proposal considers these three dimensions (Technical, human, and organizational) in a global assessment of punctual over-irradiation and under-irradiation incurred by the patients during radiotherapy and the impact of the existing safety barriers.

The main problem is the complexity of radiotherapy process, which slows the development of precise model to evaluate risk of each radiotherapy center. To avoid it, previous work focused on qualitative analysis through functional (Reitz et al. 2012-1), dysfunctional (Reitz et al. 2012-2), and organizational (Reitz et al. 2013) analysis were done. These studies proposed systematic guidelines using formalisms such as SADT or FMEA/HazOp to identify dysfunctional relations inside and between each dimension, and their impact on incurred risks (Section 3).

This paper aims to unify all these qualitative models in a probabilistic relational model (Bayesian Networks) that enables to provide not only qualitative recommendations but also a quantitative evaluation of the impact of existing barriers. Qualitative methods and structures can be applied to develop structure of a probabilistic model, which may be supplied with data from radiotherapy centers adverse events database.

\section{QUALITATIVE ANALYSIS OF RADIOTHERAPY TREATMENT PROCESS}

This section aims to present main ideas that drive our reflection to develop qualitative analysis of treatment process dysfunction through technical, human and Organizational dimensions. 


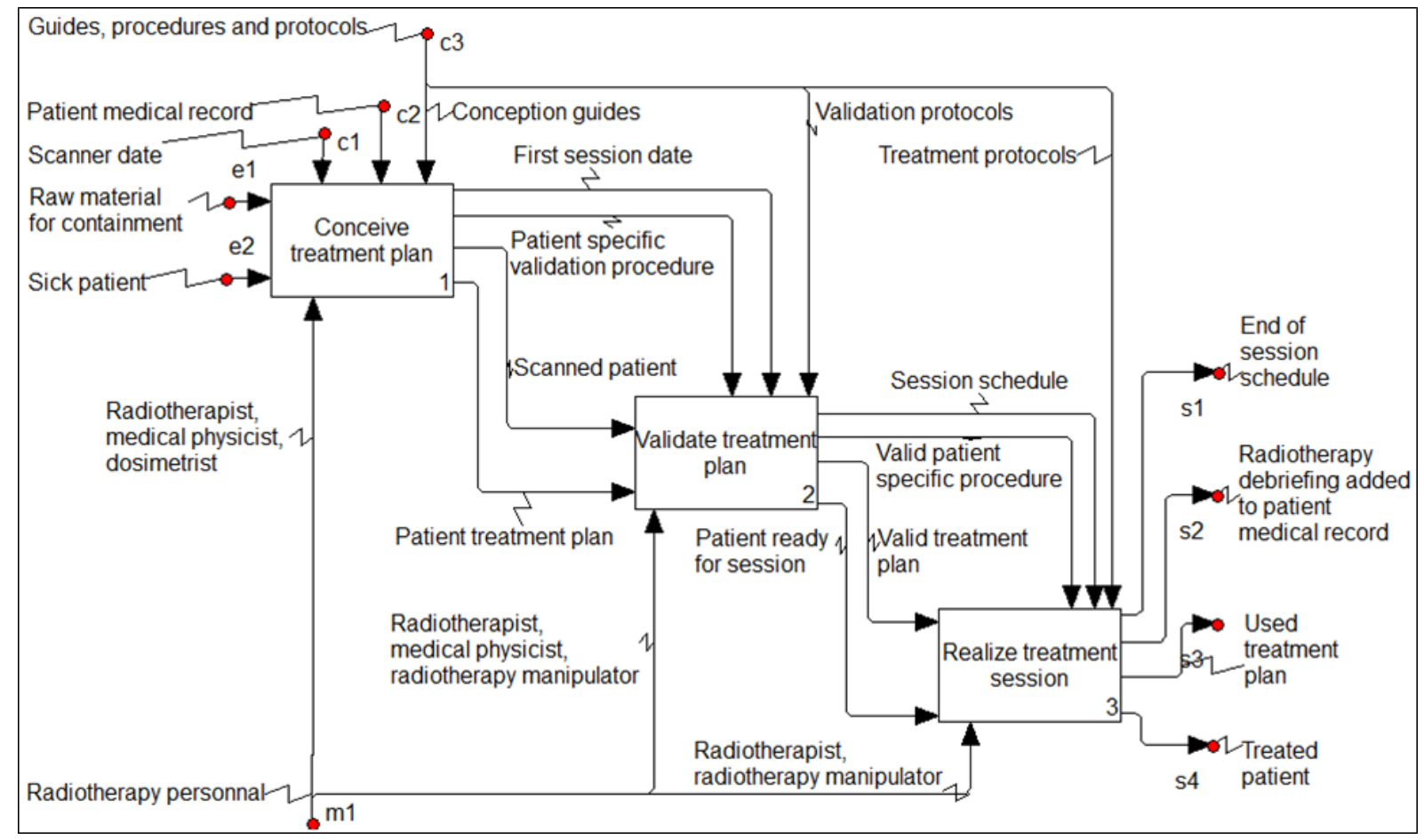

Figure 1. Highest decomposition level of the functional model of a radiotherapy treatment (SADT).

\subsection{Functional modeling: discovering the radiotherapy system objects and barriers by modeling}

To develop a precise risk model, our first study was functional modeling. To structure this modeling step, Radiotherapy is seen as the engineering process of the patient treatment plan (ISO/IEC 15288:2008). Thus, Product or system lifecycle can be used as a macro-structuration for functional modeling. In (Reitz et al. 2012-1), guidelines are proposed to develop a precise model of radiotherapy as safety barriers identification (Prepare-Perform-Close Activity sequence, Léger et al. 2009) and guidelines for object flow identification, typed as:

- Finality flow, representing patient during session, or treatment plan during design $\square$

- Technical resources, like medical devices,

- Human resources,

- $\quad$ Triggers, like dates, visa $\square$

- Organizational knowledge, like guides, protocols $\square$ made available by radiotherapy center,

- Patient particular knowledge, like medical records, or special protocols conceive for a best patient support.

Figure 1 presents the highest decomposition level of this functional model using SADT (System analysis and Design Technique).

\subsection{Dysfunctional analysis: Deductive method based on functional modeling}

From this precise functional analysis, it is possible to deduce dysfunctional analysis by systematically applying Failure Mode Effects Analysis (FMEA) on each activities and Hazard and Operability (HazOp) on object flows. Guidelines to obtain a precise dysfunctional analysis based on functional analysis have been proposed in ( Reitz et al. 2012-2) and can be resumed at:

- Activities failure can be Out of service or degraded. Each activity failure can be caused by any of its input object flows deviation, or by an internal failure. Each activity failure has consequence on any of its output object flows deviation.

- Each Input/output object flows deviation can be $\square$ more than $\square$, less than $\square$, $\square$ other than $\square \square$ is not $\square$ Those deviations concerns object properties (form, space, time) or flow properties (rank, headcount, debit).

For example: two patients with the same name are waiting for the beginning of their treatment sessions. This situation can lead to treat the wrong patient. That can be expressed by the typed deviation: $\square$ More than $\square$ debit $\square$ p patient flow $\square$. This deviation can lead to a degraded failure mode of the activity $\square$ realize treatment session $\square$ treating wrong patient. This degraded mode of the activity realize treatment session $\square$ generates the accidental situation studied: over irradiation of healthy tissues ( $[$ more than_dose_patient $₫$ healthy tissues $\square$ ), and under irradiation of tumors ( $\square$ less than_dose_patient $₫$ s tumors $\square$ ). 


\subsection{Human and Organizational analysis: knowledge extraction from French mandatory risk analysis guide}

In 2009, ASN provides a risk analysis guide designed for radiotherapy centers, to help them to evaluate their vulnerability towards a set of risks scenarios presented as FMEA tables. In this guide, 24 organizational and human factors scenarios are proposed for evaluation. The content of those scenarios have been modeled in a previous studies (Reitz et al. 2013). Figure 2 presents a sample of the models obtained. Those models present pathogenic influences between organizational, human and technical dimensions. Human and technical dimensions in those models are common with flows deviations or activities failure modes used in our previous qualitative analysis.

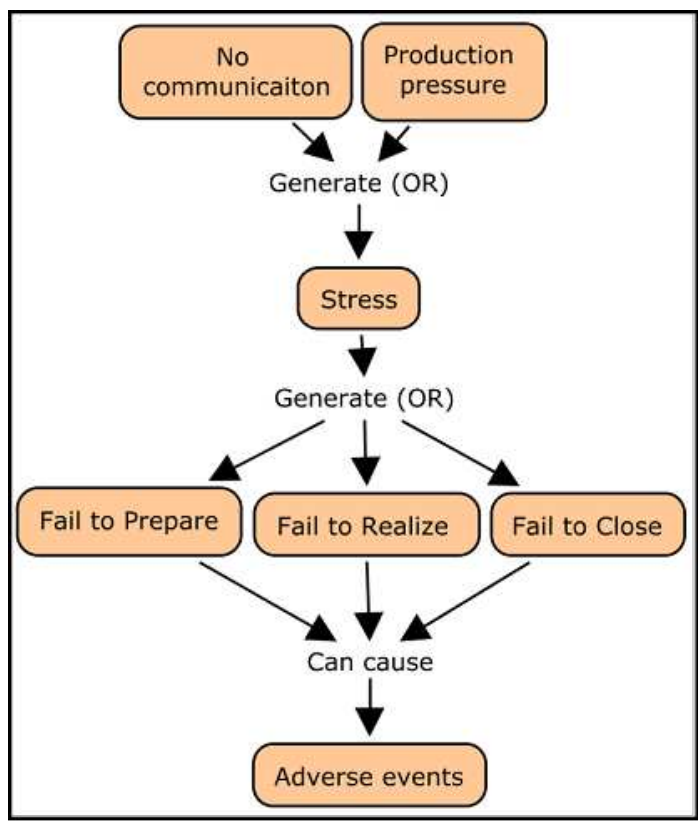

Figure 2. Sample of organizational and human pathogenic influence on technical radiotherapy process.

\section{MODELING APPROACH}

\subsection{General principles}

Based on this qualitative analysis, this paper will be centered on technical dimension of radiotherapy (Functional model and Dysfunctional analysis). Human and organizational factors will be integrated to our quantitative model in a next study. To obtain a quantitative model representing technical event chains leading to over/under-irradiation of the patient, a formalism using tree diagram will be a good choice. One of the most widespread tree formalism used in dependability allowing evaluation of risk is Fault tree (FT). But Fault tree aren $t$ fully adapted to our problem:

- FT use logic AND and OR gates to represent and evaluate risks. It is particularly adapted to represent Technical dimensions, but this method isn $t$ adapted to human and organizational factors, which can $t$ be represented with logic doors. For example, a stressed person won $t$ do systematically errors, but will certainly do more errors than if he isn $t$ stressed. That kind of behavior can $t$ be represented with binary events, conditional probability best fits with it.

- To the best of our knowledge, Automatic learning of databases isn tt possible with fault tree.

- Using fault tree on huge complex systems like radiotherapy will result in a huge fault tree, with multiple occurrence branches. That lack of concision of fault tree may be a turn off for practitioners who will have to quickly appropriate and use the model.

Bayesian Networks addresses all those problems. But, there is no structured method to develop a risk model with Bayesian network. In this paper, we propose a method adapted from FTA to develop a Bayesian Network (BN) structure from functional and dysfunctional analysis. Conditional probabilities, for the technical dimension, will be similar to logic gates AND and OR. Organizational and human factors will be integrated to the BN in a next study. 


\subsection{From functional model and dysfunctional analysis to Risk centered Bayesian network}

$\mathrm{BN}$ are defined by a directed oriented acyclic graph in which the nodes represent the system variables and the arcs symbolize the dependencies or the cause-effect relationships among the variables. A BN is defined by a set of nodes and a set of directed arcs. A probability is associated to each state of the node. This probability is defined, a priori for a root node, and computed by inference [from the content of Conditional Probability Tables(CPT)] for the others. $\square($ Jensen 1996; Weber et al. 2012). In this study, we propose a method based on fault tree analysis (FTA, Limnios 2005, Bobbio et al. 2001).

The first step of the FTA concerns preliminary qualitative analysis of the system, which are covered by functional modeling and dysfunctional analysis. The second step concerns analysis specifications, assumptions and initial conditions on the studied system: In this study, the undesirable top event chosen is the wrong irradiation of the patient (encompassing both risks explained in section 2). This event is a deviation of the flows $\square$ patient ready for session $\square$ and treated patient $\square$ in functional model and in the dysfunctional analysis. Before beginning BN construction step, some basic assumptions are made:

- Events are binary: working/ failure for activities, correct/ wrong for flows.

- Events are statistically independent

- Relationships between events and causes are represented by conditional probability tables (CPT) behaving like logic gates AND and OR (presented in figure 3).

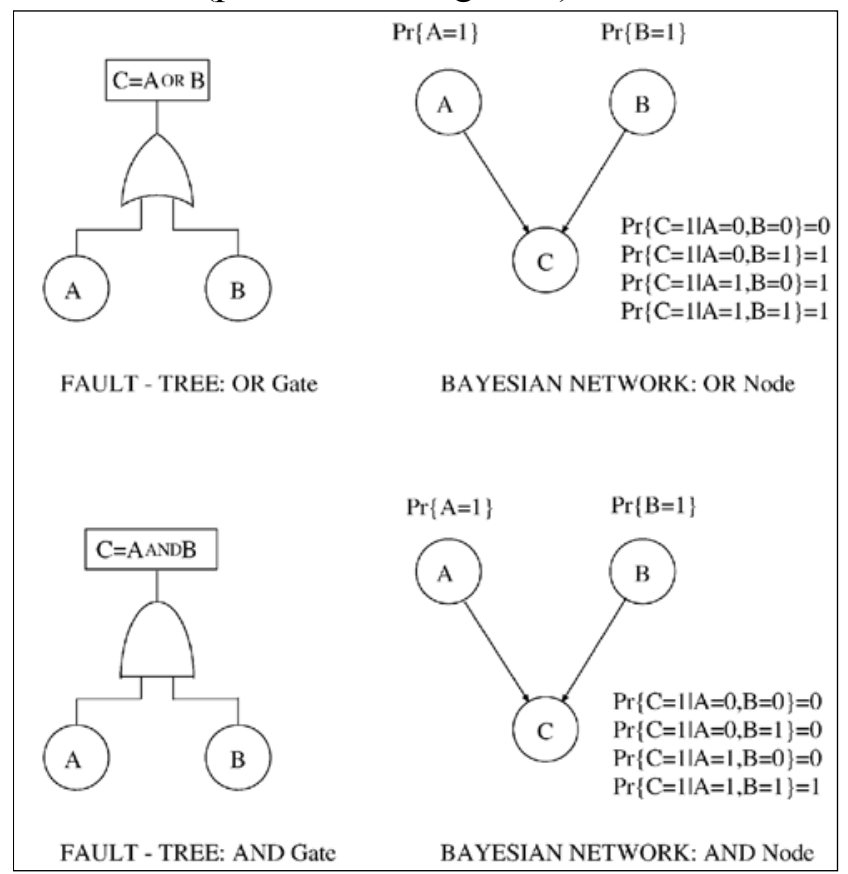

Figure 3. Equivalences between fault tree gates and Bayesian networks (Bobbio et al., 2001).

Finally, the construction step is iterative, based on the previous qualitative analysis, and can be proceeding as follows:

- Identify all the possible direct causes of the studied event on flows or activities of the functional model. For the top event wrong irradiation of the patient, the two causes are a wrong irradiation of patient out of the treatment plan validation ( $\square$ Patient ready for session $\square$ flow deviation) or out of treatment session ( $\square$ Patient treated $\square$ flow deviation).

- Identify the relationship or logic of the cause-effects events. In our case, the relationship is a logic OR.

- Structure the BN with these events and logic gate, by representing the flow or activity identified with a node, and two states on the node: Normal and failure (for activities) or wrong (for flows).

- Connect with an arrow the causes to the event, and fill in the CPT with the content of relationship previously identified.

- Keep looking back to ensure identified causes are not repeated. If so, be sure that the identified deviation of the flow (or failure of the activity) is strictly identical in both cases. If they aren $t$ dysfunctionally $\square$ identic, then duplicate events and specify the deviation of each node. If they are dysfunctionally $\square$ identic; then place an arch between that cause and the studied event. In our example, $\square$ patient ready for session $\square$ is an input flow for treatment session, and the output flow of treatment plan validation. They are duplicated on the BN because their deviations are different: in one case is represented 
the fact that the irradiation of the patient after treatment plan validation $\square$ is wrong, in the other case is represented the fact that the identity of the patient is wrong before Treatment session $\square$ (see figure 4).

- Repeat the process for the causes previously identified by searching on flows or activities directly connected to the identified causes: in this study, the identified causes are patient ready for session and treated patient $\square$. Then, the next causes we will search for concerns activities called treatment session $\square$ and treatment plan validation $\square$

Figure 4 present the BN obtained with the method presented above, applied on the functional model (and its dysfunctional analysis) presented figure 1. Each BN presented in this study have been constructing with BayesiaLab (property of Bayesia).

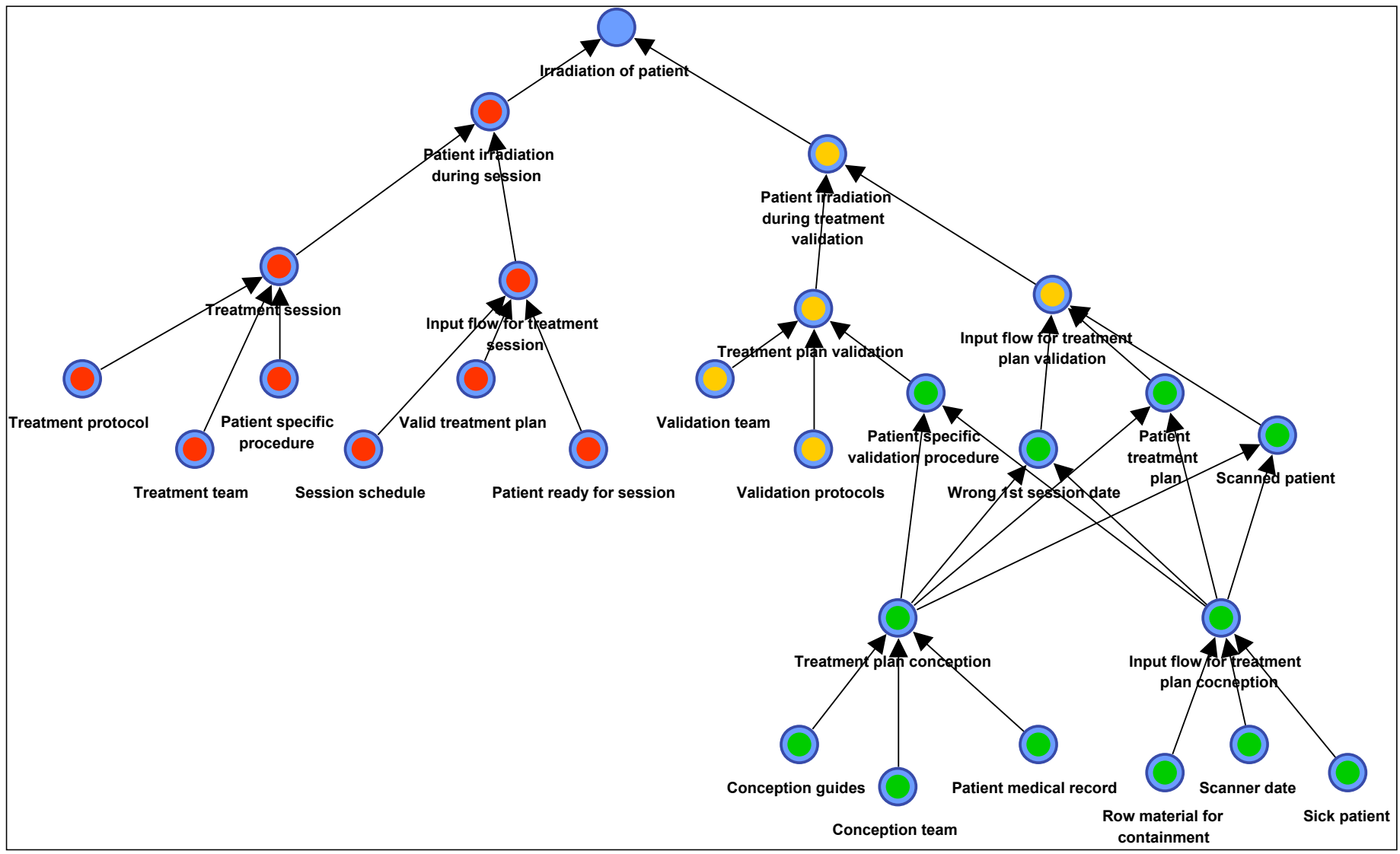

Figure 4. Bayesian network model of a wrong irradiation of patient in radiotherapy.

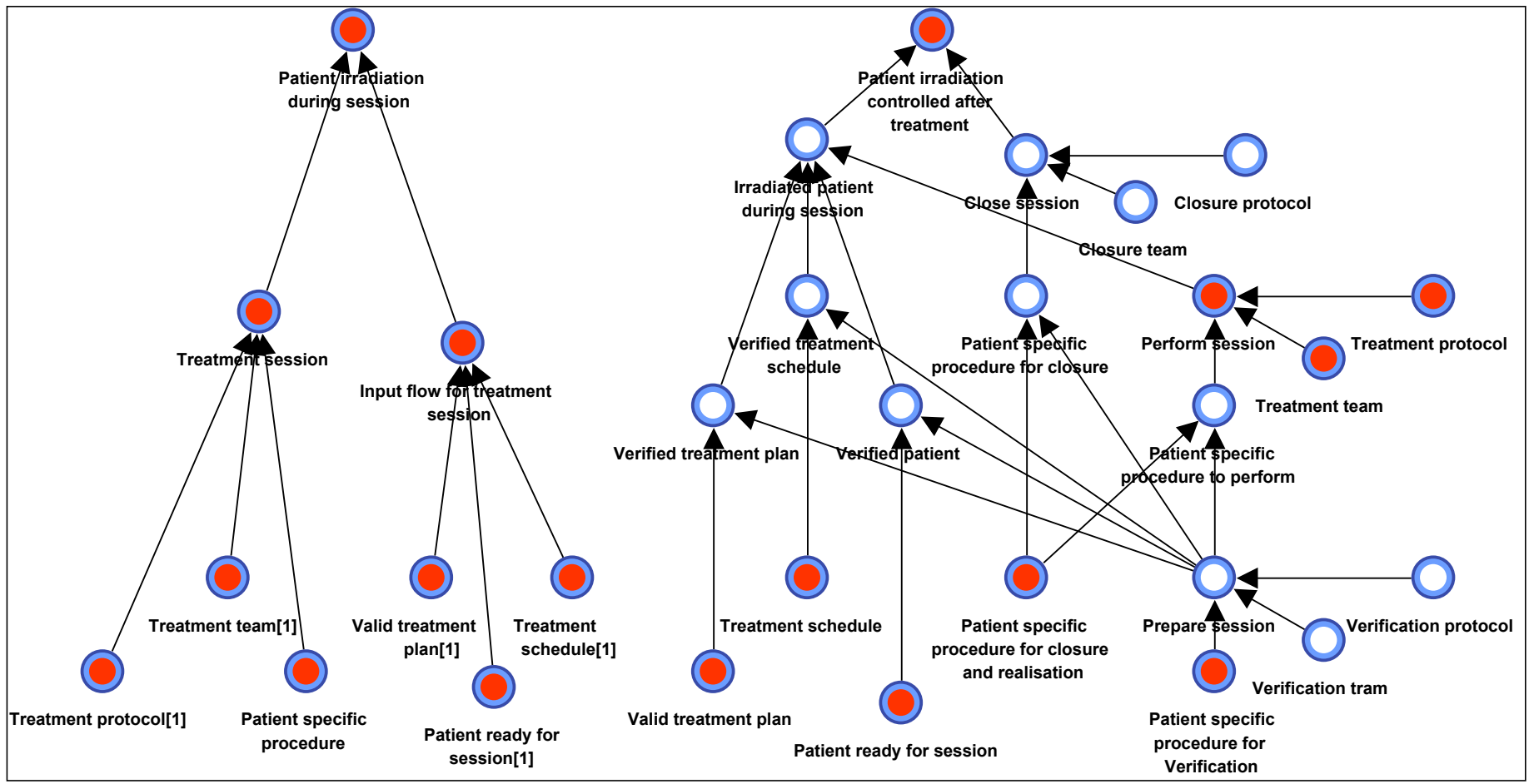

Figure 5. integration of Preventive and protective barriers before and after treatment session. 


\subsection{Modeling barriers with Bayesian network}

In the BN model proposed above, barriers are not modeled. During functional modeling, one of the barrier identification method used concerns Prepare-Perform-Close (P-P-C) activity sequence, presented as a modeling pattern to identify existing barriers on the treatment process. Structurally, PPC sequence represents the systematic verifications that must be proceeding on input flows before performing activity (Systematic Prevention barrier), and the controls done on output flows after each performing activity (Systematic Protection barrier). In this study, the activity treatment session $\square$ has been modeled with preparation and closure activities (Figure 5). Same kind of study will be done later with other kind of barriers identified during functional modeling.

To model those activities, dysfunctional behavior of verifications and controls must be exposed: a deviation on a prepared/closed input flow can entail a deviation on these output flows only if the preparation/closure is in a failure mode. That dysfunctional behavior has been related in the model by filling in CPT of each output nodes of the activities Prepare session $\square$ and $\square$ close session $\square$ with AND nodes.

The last model developed in this study is represented Figure 6 shows the final model with the PRC sequence replacing treatment session in the $\mathrm{BN}$ of figure 5

\section{FIRST QUANTITATIVE ANALYSIS AND COMPARED RESULTS}

\subsection{Data sets}

In order to make a comparison between these two BN, representing radiotherapy without barriers (Figure 4 ) and with preventive and protective barriers (Figure 6), these models requires prior probabilities to determine each parent nodes of the model. To do so, we propose a fictive data set representing a fictive center where:

- Patient flow is important,

- Human resources are over-demanded,

- Good quality of care (good knowledge, good technical resources)

To represent this fictive center the following data set address to each flow type the following prior probabilities:

- Parent nodes representing finality (sick patient, treatment plan, and patient ready for session) wrong state probability: $2 \%$

- Parent nodes representing technical resources (Row material for containment, Scanned patient and Valid treatment plan) wrong state probability: $0,01 \%$

- Parent nodes representing human resources (each teams) wrong state: $15 \%$

- Parent nodes representing triggers (dates and schedules) wrong state probability: $0,01 \%$

- Parent nodes representing organizational knowledge (guides, protocols) wrong state : $0,01 \%$

- Parent nodes representing patient particular knowledge (particular protocols and procedures) wrong state : $0,1 \%$

\subsection{Compared results}

Without barriers (Figure 4), the BN calculation gave the probability of a punctual wrong irradiation of patient occurring in $43.59 \%$. Probability of a wrong patient irradiation during treatment validation occurs in $29.41 \%$, and during treatment session the probability is $20.09 \%$.

With structural barriers during treatment session (Figure 6) global risk probability falls is lowered by $12.53 \%$ (BN Figure 6), mainly caused by a wrong irradiation during treatment plan validation (29.41\%, same calculation by each $\mathrm{BN}$ ). The $\mathrm{BN}$ calculation gave the probability of a wrong patient irradiation during treatment session at $2.33 \%$ after closure activity. After preparation and realization, this probability is $15.36 \%$. These two BN gives significantly different values.

The advantage of Bayesian network is to propose the possibility, for users, to see the impact of a known state on a node. For example: on both model, we observe that we have the wrong patient ready for session (by setting patient ready for session 100\% wrong). With the BN Figure 4, wrong patient irradiation probability is $100 \%$. In BN figure 6 , The probability to have a wrong patient after preparation step is $15.17 \%$, The probability to wrongly irradiate patient during treatment session grow up to $27.97 \%$, finally, after closure, the probability not to have detected this wrong irradiation falls to $4.23 \%$. 


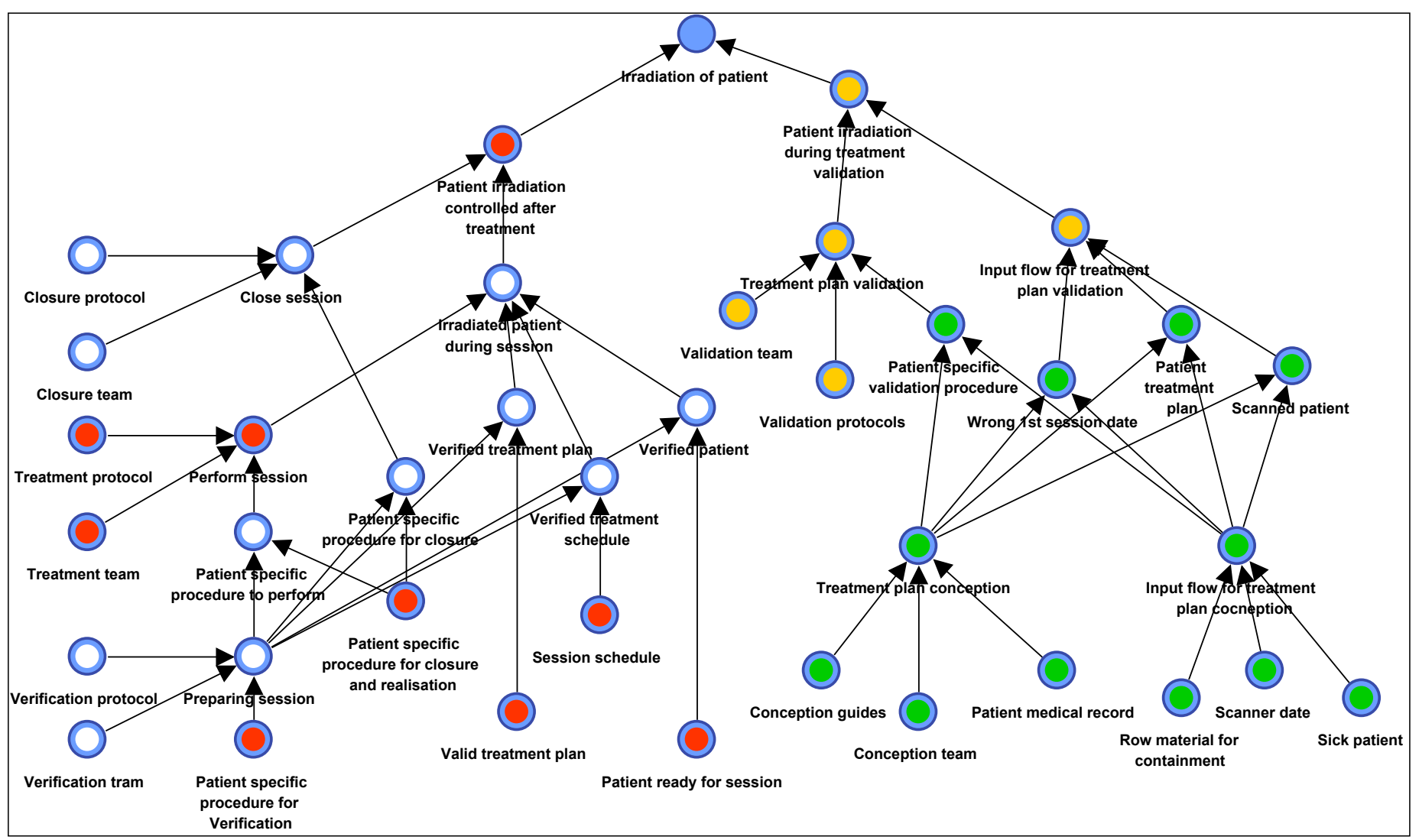

Figure 6. Bayesian network model of a wrong patient irradiation in radiotherapy, with safety barriers placed around treatment session.

\section{CONCLUSION}

The example used in this study is a macroscopic representation of radiotherapy. However, the impact of structural barriers on patient $\$$ risk has been highlighted with Bayesian Networks. This study is based on a fictive data set. This first evaluation would be more powerful by:

- Applying this method on more precise part of radiotherapy process. Then, states of each nodes will be mere relevant, but assumptions concerning the binary states could be revised.

- Connecting this model to radiotherapy centers adverse events database. Then prior probabilities, conditional probability tables, and top event evaluation should be more relevant (because based on real declared events).

By using this method in radiotherapy centers practitioners will be able, by comparison between different barriers, to see what barrier is correctly realized or overstepped, effective or not, etc.

The obtained model will be enriched with models of organizational and human influences, to obtain a global risk assessment and with other structural barriers.

\section{BIBLIOGRAPHY}

Aloui, Saber. 2008. «Contribution to the modeling and analysis of risk in a healthcare organization through a system approach ». In French. Paris: École Nationale Supérieure des Mines de Paris.

Birraux, M. Claude. 2013. "Means of Reporting and codification of criteria for significant events in the field of radiation protection excluding nuclear installations and transport of materials radioactive ». In French. Guide 11. ASN.

Bobbio, A., L. Portinale, M. Minichino, et E. Ciancamerla. 2001. «Improving the Analysis of Dependable Systems by Mapping Fault Trees into Bayesian Networks ». Reliability Engineering and System Safety 71: $249-260$.

French Health high authority - HAS. 2013. « Radiation protection of the patient and analysis of DPC practices and certification of health institutions ». In French. Guide HAS, ASN.

«French Nuclear Safety Agency - ASN ». 2013. http://www.asn.fr/. 
Holmberg, Ola. 2012. «International Reporting Systems: Safron and ROSIS » presented in Radiation Oncology Safety Workshop, september 2011, Australia.

INCa. 2013. " National Observatory of radiation survey report: the situation end of 2011 and changes since $2007 \gg$.French national cancer institute.

ISO/IEC 15288:2008. « Systems and Software Engineering -- System Life Cycle Processes ». Norm ISO/IEC 15288:2008. ISO/IEC.

Jensen, Finn V. 1996. An introduction to Bayesian networks. New York: Springer.

Léger, A., P. Weber, E. Levrat, C. Duval, R. Farret, et B. Iung. 2009. « Methodological developments for probabilistic risk analyses of socio-technical systems ». Proceedings of the Institution of Mechanical Engineers, Part O: Journal of Risk and Reliability 223 (4): $313 \square 332$.

Limnios, Nikolaos. 2005. Fault Tree. Paris: Hermès Science publications : Lavoisier.

« Radiation Oncology Safety Information System ». 2013. http://www.rosis.info/.

Reitz, A., E. Levrat, J.-F. Pétin, A. Noël, D. Aigle, D. Peiffert, et P. Graff. 2012-2. « Systematic approach for dysfunctional analysis in radiotherapy ». In French. Cancer/Radiothérapie 16 (8) (décembre): 667 673. doi:10.1016/j.canrad.2012.06.004.

Reitz, A., J.-F. Pétin, E. Levrat, A. Noel, D. Aigle, D. Peiffert, et P. Graff. 2012-1. « Functional modeling for risk analysis in radiotherapy ». In French. Cancer/Radiothérapie 16 (8) (décembre): 653-660. doi:10.1016/j.canrad.2012.06.006.

Reitz, Alexandre, Eric Levrat, et Jean-François Pétin. 2013. «Organizational and Human factors for risk analysis in Radiotherapy ». In French. Qualita 2013, in Compiègne, France.

Weber, P., G. Medina-Oliva, C. Simon, et B. Iung. 2012. «Overview on Bayesian networks applications for dependability, risk analysis and maintenance areas ». Engineering Applications of Artificial Intelligence 25 (4) (juin): 671-682. doi:10.1016/j.engappai.2010.06.002.

World Health Organisation. 2008. « Radiotherapy Risk Profile ». Technical Manual WHO/IER/PSP/2008.12. World Health Organisation. 